\title{
Spectral properties of Andreev crystals
}

\author{
Mikel Rouco $\odot,{ }^{1,{ }^{*}}$ F. Sebastian Bergeret $\odot,{ }^{1,2, \dagger}$ and Ilya V. Tokatly $\odot^{2,3,4,5, \ddagger}$ \\ ${ }^{1}$ Centro de Física de Materiales (CFM-MPC), Centro Mixto CSIC-UPV/EHU, Manuel de Lardizabal 5, E-20018 San Sebastián, Spain \\ ${ }^{2}$ Donostia International Physics Center (DIPC), Manuel de Lardizabal 4, E-20018 San Sebastian, Spain \\ ${ }^{3}$ Nano-Bio Spectroscopy group and European Theoretical Spectroscopy Facility (ETSF), Departamento de Polímeros y Materiales \\ Avanzados: Física, Química y Tecnología, Universidad del País Vasco, Av. Tolosa 72, E-20018 San Sebastián, Spain \\ ${ }^{4}$ IKERBASQUE, Basque Foundation for Science, E48009 Bilbao, Spain \\ ${ }^{5}$ ITMO University, Department of Physics and Engineering, Saint-Petersburg, Russia
}

(Received 29 April 2021; revised 29 June 2021; accepted 28 July 2021; published 13 August 2021)

\begin{abstract}
We present an exhaustive study of Andreev crystals (ACs)—quasi-one-dimensional superconducting wires with a periodic distribution of magnetic regions. The exchange field in these regions is assumed to be much smaller than the Fermi energy. Hence, the transport through the magnetic region can be described within the quasiclassical approximation. In the first part of the paper, by assuming that the separation between the magnetic regions is larger than the coherence length, we derive the effective nearest-neighbor tight-binding equations for ACs with a helical magnetic configuration. The spectrum within the gap of the host superconductor shows a pair of energy-symmetric bands. By increasing the strength of the magnetic impurities in ferromagnetic ACs, these bands cross without interacting. However, in any other helical configuration, there is a value of the magnetic strength at which the bands touch each other, forming a Dirac point. Further increase of the magnetic strength leads to a system with an inverted gap. We study junctions between ACs with inverted spectrum and show that junctions between (anti)ferromagnetic ACs (always) never exhibit bound states at the interface. In the second part, we extend our analysis beyond the nearest-neighbor approximation by solving the Eilenberger equation for infinite ACs and junctions between semi-infinite ACs with collinear magnetization. From the obtained quasiclassical Green functions, we compute the local density of states and the local spin polarization in anti- and ferromagnetic ACs. We show that these junctions may exhibit bound states at the interface and fractionalization of the surface spin polarization.
\end{abstract}

DOI: 10.1103/PhysRevB.104.064506

\section{INTRODUCTION}

Magnetic defects and regions in a superconductor may lead to bound states that strongly change the local spectrum [1-12]. When the magnetic exchange coupling is larger than the Fermi energy and the size of the magnetic region is small compared to the superconducting coherence length, a pair of non-degenerate states with opposite energies appear inside the superconducting gap. These are the so-called YuShiba-Rusinov (YSR) states [1-3]. In contrast, if the exchange coupling is small compared to the Fermi energy, $\mu$, a pair of degenerate bound states appear $[4,12,13]$. The origin of such degeneracy can be understood from a semiclassical perspective: electrons at the Fermi level traveling through the magnetic region are not back-scattered, but they accumulate a phase, $\Phi$. This phase has the opposite sign for electrons/holes and spin up/down. This results in double-degenerate bound states formed by electrons from the Fermi valleys at either $+k_{F}$ or $-k_{F}$ [see Fig. 1(b)]. In a normal metal, the phase accumulated can be gauged out. In contrast, if the host material is

\footnotetext{
*mikel.rouco@ehu.eus

†fs.bergeret@csic.es

‡ilya.tokatly@ehu.es
}

a superconductor, the Andreev reflection at the semiclassical impurities leads to the coupling between electrons and holes at the same Fermi valley. This mechanism leads to Andreev bound states inside the superconducting gap. The crossover from the YSR to Andreev limit has been studied in detail in Ref. [12].

In a periodic arrangement of magnetic impurities, as, for example, a chain, the single-impurity bound states hybridize and form bands within the superconducting gap. Those bands have been widely studied for atomic-sized magnetic impurities[14-22]. The hybridization of YSR states can lead to topological phases which host Majorana bound states at the ends of the impurity chain. In Ref. [23], we studied the analog of such atomic chains in a mesoscopic structure with lateral dimensions smaller than the superconducting coherence length, $\xi_{0} \equiv \hbar v_{F} / \Delta$ (here, $v_{F}$ is the Fermi velocity and $\Delta$ the superconducting order parameter) and replaced the magnetic impurities by semiclassical magnetic regions. [See the sketch in Fig. 1(a)]. In that work, we only considered chains with co-linear magnetization. Motivated by the appearance of a topological state in atomic chains with a rotating magnetization, we extend our previous work to the study of the spectrum of semiclassical helical chains. Moreover, we use the quasiclassical method to determine the local spectral properties of such semiclassicla crystals, which we call Andreev crystals 
(a)

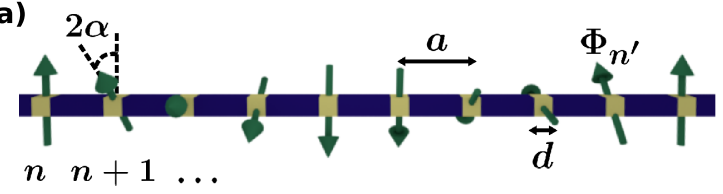

(b)

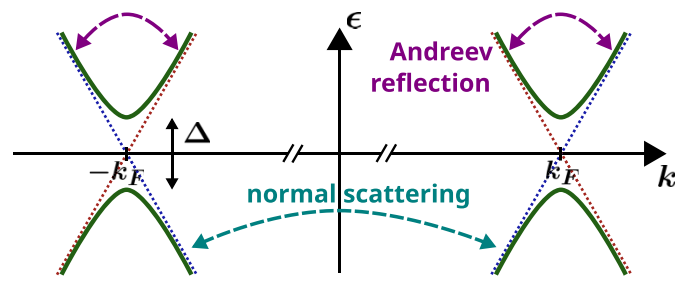

FIG. 1. (a) Sketch of a quasi-1D helical Andreev crystal formed by a superconducting wire interrupted by magnetic regions of width $d$ separated by a constant distance $a$. It is assumed that $k_{F}^{-1} \ll d \ll$ $\xi_{0}$. The magnetization of the impurities rotates an angle $2 \alpha$ around the $x$ axis between subsequent impurities and has a strength given by the magnetic phase $\Phi_{n}$ [see Eq. (1)] in the $n$th impurity. (b) Sketch of the superconducting spectrum with the two electron-hole (e-h) valleys at $\pm k_{F}$. The semiclassical impurities forming the Andreev crystal cause only small momentum transfer processes that couple quasiparticles within the same e-h valley via Andreev scattering. Because there is no normal reflection coupling quasiparticles from opposite valleys the system presents a twofold degeneracy.

(ACs). Mesoscopic structures involving superconductors and ferromagnetic materials have been extensively studied, in both the diffusive [24-31] and ballistic limit [32-35]. Our focus here is from a different perspective, more in line with those works on Shiba chains.

Specifically, in this article we present the general theory of ACs, including non-collinear magnetization orientation and arbitrary separation between the magnetic impurities. In a first part we consider chains of impurities with noncollinear magnetization where the magnetic regions are separated by a distance $a \gtrsim \xi_{0}$. We solve the nearest-neighbor tight-binding equations of helical ACs, where the exchange field rotates a constant angle $2 \alpha$ around a fixed axis between subsequent magnetic impurities, whereas their strength remains constant, $\Phi$. The spectrum of helical ACs for energies within the superconducting gap, $|\epsilon|<|\Delta|$, shows a pair of Andreev bands with symmetric energy with respect to the Fermi level. In ferromagnetic configurations $(\sin \alpha=0)$ the Andreev bands cross without interacting, closing the gap in a finite range of $\Phi$ values around half-integer values of $\Phi / \pi$. Otherwise, the Andreev bands touch each other only at half-integer values of $\Phi / \pi$ forming a Dirac point. In junctions between semi-infinite helical ACs where the rotation, $\alpha$, remains constant all along the chain and the magnetic phase changes from $\Phi_{L}$ to $\Phi_{R}$ at the left and right sides of the junction, respectively, states bounded to the interface may appear when $\operatorname{sign}\left(\tan \Phi_{L}\right) \neq$ $\operatorname{sign}\left(\tan \Phi_{R}\right)$. We refer to the junctions fulfilling this condition as inverted junctions of ACs and they maintain similarities with Dirac system with a spatial mass inversion [36-39]. We show that inverted junctions of (anti)ferromagnetic ACs (always) never support interfacial states and that the range of parameters $\Phi_{L(R)}$ for which the bound states appear increases as the rotation approaches an antiferromagnetic ordering (i.e., with decreasing value of $|\cos \alpha|)$.

In a second part, we present exact calculations of the spectral properties of (anti)ferromagnetic ACs and junctions beyond the tight-binding approximation used in previous works [23]. Specifically, we solve the Eilenberger equation and obtain the quasiclassical Green's functions (GFs) in different situations. The magnetic regions are described by effective boundary conditions that take into account the spin-dependent jump of the phase, $\Phi$. On the one hand, our solution provides the exact energy and spatial distribution of the density of states and spin polarization of the system. On the other hand, our results demonstrate the validity of the first neighbor tight-binding approximation regarding the gap closing in infinite antiferromagnetic ACs at half-integer values of $\Phi / \pi$, the appearance of a pair of states bounded to the interface between two antiferromagnetic ACs with inverted gaps, and the fractionalization of the surface spin polarization in such junctions [23].

This article is organized as follows. In Sec. II, we introduce the model Hamiltonian and the main equations used for the nearest-neighbor tight-binding model (Sec. II A), and the Eilenberger GFs (Sec. II B). In Sec. III, we solve the nearest-neighbor tight-binding equations of infinite $\mathrm{ACs}$ and junctions between semi-infinite helical ACs, i.e., ACs where the exchange field of the semiclassical impurities form an helix along the wire. In Sec. IV, we focus on ACs where the exchange field of all the impurities is collinear. In particular, we solve the Eilenberger equation to obtain the quasiclassical GF of ACs with magnetic impurities following (Sec. IV A) ferromagnetic and (Sec. IV B) antiferromagnetic ordering. In Sec. IV C, we present the method to solve the Eilenberger equation in junctions between semi-infinite collinear ACs and we apply it to obtain the quasiclassical GFs in junctions between antiferromagnetic ACs. Finally, in Sec. V, we summarize the main results of the paper.

\section{THE MODEL AND MAIN EQUATIONS}

We consider a superconducting wire of lateral dimensions much smaller than the superconducting coherence length, $\xi_{0}$. The wire contains magnetic regions located at the points $X_{n}=n a$, where $a$ is the separation between the impurities and $n$ is the impurity index. We assume that the width of the magnetic regions, $d$ is larger than $k_{F}^{-1}$ and hence can be considered within the semiclassical approach [12]. In addition, we also assume that $d \ll \xi_{0}$ such that we can treat the magnetic regions as pointlike impurities, in the semiclassical scale, with a polarization strength and direction proportional to the corresponding $\mathrm{SU}(2)$ magnetic phase [13,40],

$$
\hat{\boldsymbol{\sigma}} \cdot \boldsymbol{\Phi}_{n} \equiv \frac{1}{\hbar v_{F}} \int d x \hat{\boldsymbol{\sigma}} \cdot \boldsymbol{h}_{n}(x)
$$

Here, $v_{F}$ is the Fermi velocity, and $\boldsymbol{h}_{n}(x)$ is the exchange field vector induced by the $n$-th impurity which is assumed to be parallel to the local magnetization of the magnetic region. The Bogoliubov-de Gennes (BdG) Hamiltonian [41] describing 
the $\mathrm{AC}$ in the Andreev limit reads,

$$
\check{H}_{\mathrm{BdG}}^{\eta}(x)=-i \eta \hbar v_{F} \hat{\tau}_{3} \partial_{x}+\hat{\tau}_{1} \Delta-\hbar v_{F} \sum_{n} \hat{\boldsymbol{\sigma}} \cdot \boldsymbol{\Phi}_{n} \delta\left(x-X_{n}\right),
$$

where $\hat{\tau}_{i}$ are the Pauli matrices spanning the Nambu space (i.e., the electron-hole space), $\hat{\sigma} \equiv\left(\hat{\sigma}_{1}, \hat{\sigma}_{2}, \hat{\sigma}_{3}\right)$ stands for the vector of Pauli matrices that span the spin space and $\eta= \pm$ refers to the two electron-hole valleys at $\pm k_{F}$ [see Fig. 1(b)]. A distinctive feature of semiclassical impurities is that they do not trigger back-scattering processes. This allows us to treat the two Fermi valleys separately and to drop the $\eta$ index. In the Andreev equations [42], Eq. (3), the delta functions describe the boundary conditions within the semiclassical approach. Namely, they describe the phase gained by a quasiparticle when it traverses the magnetic region [see Eq. (6) below].

The solution of the BdG equations provides all the spectral information about the crystal. As it will be shown in Sec. II A, one can solve this problem analytically under the assumption that magnetic impurities are weakly coupled to each other, $e^{-a / \xi_{0}} \ll 1$. In this limit the system can be described by an effective tight-binding model which provide the spectrum of this system. A drawback of this approach is that to compute observable quantities, such as the local density of states or the local spin density, one has to perform explicit summation over the Bloch momentum. Indeed, for calculation of observables it is more convenient to use the quasiclassical Eilenberger equation [43]. This formalism is presented in Sec. II B. Specifically, we show how to obtain exact analytical expressions for the quasiclassical Green's functions (GFs) of periodic ACs, and how to access to observables in a rather simple way. Thus both formalisms presented in Secs. II A and II B are complementary and provides a full description of ACs. Note that the quasiclassical approach requires that the distance between the impurities to be larger than the Fermi wave length, $k_{F} a \gg 1$. Moreover, in the ferromagnetic alignment, in order to avoid the self-consistent computation of the superconducting gap, we assume that $a$ is larger (or of the same order of $\xi_{0}$ ). In the antiferromagnetic case, this restriction is relaxed due to the smaller effective exchange field.

\section{A. Tight-binding equations}

To obtain the spectral properties of an AC one needs to solve the Andreev equations,

$$
\check{H}_{\mathrm{BdG}}(x) \check{\Psi}(x)=\epsilon \check{\Psi}(x),
$$

where $\breve{H}_{\mathrm{BdG}}(x)$ is the Hamiltonian, Eq. (2), and $\breve{\Psi}(x)$ is a four-component spinor in the Nambu $\times$ spin space. The general solution of Eq. (3) in the region between two neighboring impurities, $X_{n}<x<X_{n+1}$, reads

$$
\check{\Psi}(x)=B_{n+1}^{+} e^{\frac{x-X_{n+1}}{\xi}}|+\rangle+B_{n}^{-} e^{-\frac{x-X_{n}}{\xi}}|-\rangle .
$$

Here $\xi \equiv \frac{\hbar v_{F}}{\sqrt{\Delta^{2}-\epsilon^{2}}}$ is the energy-dependent superconducting coherence length, $B_{n}^{+(-)}$is a two-component spinor (covering the spin space) that contains the amplitudes of the contributions to the wave function that decays from the $n$th impurity into the left (right), and

$$
| \pm\rangle \equiv \frac{e^{ \pm i \theta / 2}}{\sqrt{2 \cos \theta}}\left(\begin{array}{c}
1 \\
\pm i e^{\mp i \theta}
\end{array}\right),
$$

are two-component spinors in the Nambu space, where $e^{i \theta} \equiv$ $\frac{\sqrt{\Delta^{2}-\epsilon^{2}}+i \epsilon}{\Delta}$ is the Andreev factor. Direct product is assumed between the spinors in Nambu and spin spaces.

Within the semiclassical limit, quasiparticles traveling through the $n$th semiclassical impurity do not back-scatter, but pick up a phase according to

$$
\check{\Psi}\left(X_{n}^{R}\right)=e^{i \hat{\tau}_{3} \hat{\sigma} \cdot \Phi_{n}} \check{\Psi}\left(X_{n}^{L}\right)
$$

because of $\hat{\tau}_{3}$ and $\hat{\boldsymbol{\sigma}} \cdot \boldsymbol{\Phi}_{n}$, the sign of the accumulated phase is different for electron/holes and spin up/down quasiparticles along the exchange field direction, respectively. Applying these boundary conditions to the general wave function in Eq. (4) we obtain the equations for the $B^{ \pm}$coefficients, which can be recast into an effective tight-binding model by keeping terms up to first order in $e^{-a / \xi}$. In particular, in the limit where $e^{-a / \xi} \ll 1$, coefficients $B_{n}^{-}$at each site $n$ can be related to their counterparts, $B_{n}^{+}$, as follows:

$$
B_{n}^{-}=i \hat{\boldsymbol{\sigma}}_{n} \frac{\Delta \sin \Phi_{n}}{\sqrt{\Delta^{2}-\epsilon^{2}}} B_{n}^{+},
$$

where $\Phi_{n}=\left|\Phi_{n}\right|$ is the strength of the magnetic phase vector, and we define $\hat{\boldsymbol{\sigma}}_{n} \equiv \frac{\hat{\sigma} \cdot \Phi_{n}}{\Phi_{n}}$. It is convenient to introduce the rescaled coefficients, $b_{n}^{\prime} \equiv \hat{\sigma}_{n} \sin \Phi_{n} B_{n}^{+}$, which satisfy a tightbinding-like equation

$$
\left(\omega-\hat{\boldsymbol{\sigma}}_{n} \omega_{0 n}\right) b_{n}^{\prime}=\hat{\boldsymbol{\sigma}}_{n+1} t_{n+1} b_{n+1}^{\prime}+\hat{\boldsymbol{\sigma}}_{n} t_{n} b_{n-1}^{\prime} .
$$

Here $\omega \equiv \frac{\epsilon}{\sqrt{\Delta^{2}-\epsilon^{2}}}, \hat{t}_{n} \equiv-\frac{e^{-a / \xi}}{\sin \Phi_{n}}$ is the hopping amplitude, and $\omega_{0 n}=\frac{\cos \Phi_{n}}{\sin \Phi_{n}}$ is the value of the function $\omega$ evaluated at the bound state energy in the $n$-th impurity, $\epsilon_{0 n}=\frac{\left|\sin \Phi_{n}\right|}{\tan \Phi_{n}}$. In principle, Eq. (8) describes an arbitrary AC with lattice constant $a$. In Ref. [23], it was solved for collinear magnetization of the impurities. In Sec. III, we analyze helical ACs composed by identical magnetic impurities with an spatially rotating magnetization, forming a helix in the $y-z$ plane.

\section{B. Eilenberger equation}

Because of its simplicity, the tight-binding formulation, Eq. (8), is very useful for describing the spectral properties of ACs. However, one should bear in mind that it has been derived within first-neighbor approximation, and therefore it is valid as long as $e^{a / \xi} \ll 1$. To go beyond this approximation, we introduce here the Eilenberger equation [43] from which we can determine the quasiclassical Green's functions (GFs).

We focus again on pointlike semiclassical magnetic impurities. The Eilenberger equation in the regions between the impurities has a simple form:

$$
\hbar v_{F} \partial_{x} \breve{g}(x)-\left[i \epsilon \hat{\tau}_{3}+\Delta \hat{\tau}_{2}, \breve{g}(x)\right]=0 .
$$

Here $\check{g}(x)$ is the quasiclassical Green's function (GF), which is a $4 \times 4$ matrix in the Nambu $\times$ spin space that satisfies the normalization condition, $\breve{g}^{2}=1$. The square brackets stand for the commutation operation. $\Delta$ is the superconducting gap, which is assumed to be constant along the superconducting 
wire. Solving Eq. (9), we obtain the propagation of the GF along the superconducting region,

$$
\check{g}(x)=\hat{u}\left(x-x_{0}\right) \check{g}\left(x_{0}\right) \hat{u}\left(x_{0}-x\right),
$$

where the propagator reads

$$
\hat{u}\left(x-x_{0}\right)=\hat{P}_{+} e^{\left(x-x_{0}\right) / \xi}+\hat{P}_{-} e^{-\left(x-x_{0}\right) / \xi} .
$$

Here $\hat{P}_{ \pm} \equiv| \pm\rangle\langle\mathcal{I}|=\frac{e^{ \pm i \hat{t}_{3} \theta} \pm \hat{\tau}_{2}}{2 \cos \theta}$ are two orthogonal projectors that span the Nambu space, $| \pm\rangle$ are the basis column vectors of Eq. (5), and

$$
\langle\underline{ \pm}| \equiv \frac{e^{ \pm i \theta / 2}}{\sqrt{2 \cos \theta}}\left(1 \quad \mp i e^{\mp \theta}\right),
$$

are the co-basis row vectors orthonormal to $| \pm\rangle$. The inverse of the propagator in $\mathrm{Eq}(11)$ fulfills the relation $[\hat{u}(\tilde{x})]^{-1}=$ $\hat{u}(-\tilde{x})$.

Additionally, the GF at the right and left sides of the $n$th semiclassical impurity $\left(X_{n}^{R}\right.$ and $X_{n}^{L}$, respectively) are connected by a propagationlike boundary conditions,

$$
\check{g}\left(X_{n}^{R}\right)=e^{i \hat{\tau}_{3} \hat{\sigma} \cdot \Phi_{n}} \check{g}\left(X_{n}^{L}\right) e^{-i \hat{\tau}_{3} \hat{\sigma} \cdot \Phi_{n}} .
$$

This expression together with Eq. (11), determines the GF at any space point provided its value at a given point, $\breve{g}\left(x_{0}\right)$.

In an infinite periodic ACs we need to match the value of the GF at equivalent points of different unit cells. For this sake, it is useful to introduce the chain propagator, $\breve{S}$, that describes the propagation of the quasiclassical GF from a given position inside a unit cell to the equivalent position in the subsequent unit cell, $\breve{g}\left(x_{0}+l\right)=\breve{S} \breve{g}\left(x_{0}\right) \breve{S}^{-1}$ (here $l$ denotes the length of the unit cell). The exact form of $\check{S}$ depends on the arrange of impurities and the choice of the initial point inside the unit cell, $x_{0}$. Here we choose for $x_{0}$ the left interface of one of the magnetic impurities. Thus the chain propagator reads

$$
\breve{S} \equiv \prod_{j=1}^{J} \hat{u}(a) e^{i \hat{\imath}_{3} \hat{\sigma} \cdot \Phi_{j}},
$$

where $J$ is the number of impurities forming the unit cell. The value of the quasiclassical GF at $x_{0}$ is obtained from the periodicity along the unit cell, $\check{g}\left(x_{0}\right)=\check{S} \breve{g}\left(x_{0}\right) \check{S}^{-1}$, together with the normalization condition, $\left[\check{g}\left(x_{0}\right)\right]^{2}=1$. Once $\breve{g}\left(x_{0}\right)$ is determined the full quasiclassical GF, $\breve{g}(x)$, is obtained after propagation using Eqs. (11) and (13).

From the knowledge of the GF, we can obtain the local density of states (LDOS),

$$
v(x, \epsilon)=\operatorname{Re}\left\{\frac{1}{4} \operatorname{Tr}\left[\hat{\tau}_{3} \check{g}(x, \epsilon)\right]\right\},
$$

and the local spin density,

$$
s(x, \epsilon)=\frac{\hbar}{2} \operatorname{Re}\left\{\frac{1}{4} \operatorname{Tr}\left[\hat{\sigma}_{3} \hat{\tau}_{3} \check{g}(x, \epsilon)\right]\right\},
$$

where the traces run over the Nambu $\times$ spin space. In Sec. IV, we use this approach to obtain the quasiclassical GFs of ferromagnetic and antiferromagnetic ACs and we generalized this method to study junctions between different (anti)ferromagnetic ACs.

\section{HELICAL ANDREEV CRYSTALS}

In this section, we study the spectral properties of ACs with a periodic rotation of the magnetization of the magnetic impurities. For this sake, we use the tight-binding approach introduced in Sec. II A. In particular, we focus on an AC consisting of identical magnetic impurities whose magnetization is in the $y-z$ plane and rotates by a constant angle, $2 \alpha$ around the $x$ axis $^{1}$ [see Fig. 1(a)]. The SU(2) magnetic phase describing this situation is given by

$$
\hat{\boldsymbol{\sigma}}_{n} \cdot \boldsymbol{\Phi}_{n}=\Phi e^{-i \hat{\sigma}_{1} \alpha n} \hat{\sigma}_{3} e^{i \hat{\sigma}_{1} \alpha n},
$$

where $\Phi$ is the strength of the magnetic phase. Its strength is the same in all the impurities. Substituting this expression into Eq. (8), we obtain that

$$
\left[\omega-e^{i \hat{\sigma}_{1} \alpha} \hat{\sigma}_{3} \omega_{0}\right] b_{n}=\hat{\sigma}_{3} t\left(b_{n+1}+b_{n-1}\right),
$$

where we have defined the coefficients $b_{n} \equiv e^{i \hat{\sigma}_{1} \alpha\left(n+\frac{1}{2}\right)} b_{n}^{\prime}$. Here $\omega_{0}=\frac{\cos \Phi}{\sin \Phi}$ stands for the energy of the single-impurity levels and $t=-\frac{e^{-a / 5}}{\sin \Phi}$ is the hopping amplitude. Note the hopping amplitude is energy dependent through the energydependent superconducting coherence length, $\xi$, defined below Eq. (4). After this redefinition of the coefficients, Eq. (18) reduces to the typical tight-binding system of identical equations, whose solution reads $b_{n}=b e^{i k n a}$ and $\omega=$ $\pm \sqrt{\omega_{0}^{2} \sin ^{2} \alpha+\left(\omega_{0} \cos \alpha+2 t \cos k a\right)^{2}}$. Here, $k$ is the Bloch momentum, and the spinors $b$ are obtained from Eq. (18). The Andreev bands are defined by

$$
\frac{\epsilon}{\Delta}= \pm \sqrt{\frac{\omega_{0}^{2} \sin ^{2} \alpha+\left(\omega_{0} \cos \alpha+2 t \cos k a\right)^{2}}{1+\omega_{0}^{2} \sin ^{2} \alpha+\left(\omega_{0} \cos \alpha+2 t \cos k a\right)^{2}}},
$$

where $t$ has to be evaluated at the energy of the single-impurity level $\omega_{0}$. In Figs. 2(b) and 2(c), we show the subgap spectrum of ACs with different values of $\Phi$ and $\alpha$. At $\Phi=0$, no bound states appear, and hence there are no Andreev bands. Increasing $\Phi$, a pair of bands emerge from the coherent peaks and start moving towards the Fermi level, up to a point around $\Phi=\pi / 2$ where they touch each other, forming a gapless phase. Further increase of $\Phi$ leads to a gap reopening with inverted Andreev bands. The latter merge with the continuum spectrum at $\Phi=\pi$. Interestingly, the bands' inversion also happens when they merge into the continuum and reenter the superconducting gap at $\Phi=l \pi$, where $l$ is an integer. Consequently, the spectrum of these ACs is $\pi$ periodic in $\Phi$.

As can be seen from the energy spectrum of the bands, Eq. (19), the gap closes only at half-integer values of $\Phi / 2$ forming a Dirac point at $k a=\pi / 2$ in ACs with any value of $\alpha$ except in those where $\sin \alpha=0$. This situation corresponds to ferromagnetic ACs, where each of the Andreev bands corresponds to opposite spin species, and hence they do not interact while crossing.

In Ref. [23], it was shown that a junction between two antiferromagnetic ACs with inverted gaps presents states bounded do the interface. This corresponds to the situation where

\footnotetext{
${ }^{1}$ Because we do not include any spin-orbit interaction in our analysis any other planar rotation choice will give equivalent results.
} 
(a)

(b)
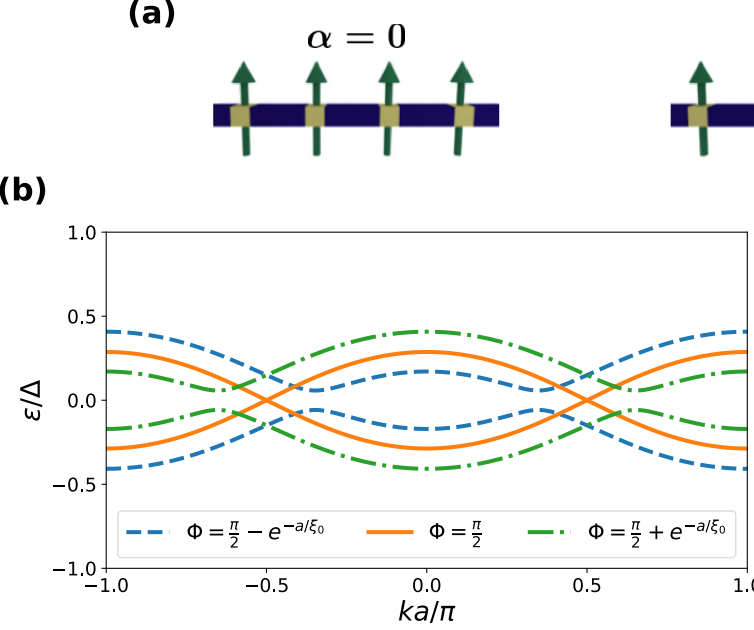

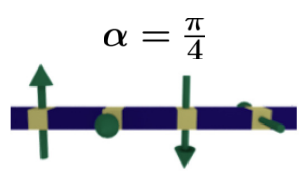

(c)
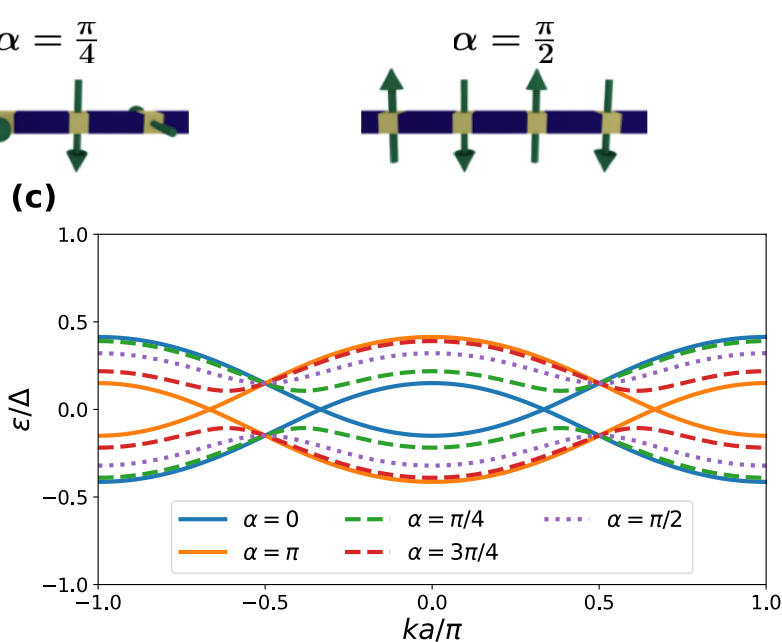

FIG. 2. (a) Sketches of magnetic configurations in ACs for different values of $\alpha$. (b) Andreev bands for $\alpha=\frac{\pi}{4}$ and different values of $\Phi$. (c) Andreev bands for $\Phi=\frac{\pi}{2}-e^{-a / \xi_{0}}$ and different values of $\alpha$. In both panels, we assumed a separation between impurities of $a=2 \xi_{0}$.

$\cos \alpha=0$ all along the structure and the sign of $\omega_{0}$ changes across the junction. It becomes interesting, then, to study if those bound states survive for arbitrary values of $\alpha$. To do so, we consider a junction between two different chains where the rotation parameter between the impurities remains constant $\alpha$, but their magnetic phases change from the AC on the left, $\Phi_{L}$, to the one on the right $\Phi_{R}$. The tight-binding equations of such a system read

$$
\left[\omega-e^{i \hat{\sigma}_{1} \alpha} \hat{\sigma}_{3} \omega_{0 n}\right] b_{n}=\hat{\sigma}_{3} t_{n+1} b_{n+1}+\hat{\sigma}_{3} t_{n} b_{n-1},
$$

where $\omega_{0 n}$ and $t_{n}$ are defined below Eq. (8). The magnetic phase is $\Phi_{L}$ for $n<0$ and $\Phi_{R}$ for $n \geqslant 0$. We can write for the left and right ACs, $b_{n}=b_{L+} e^{-i q_{L_{+} n}}+b_{L-} e^{-i \mu_{L} q_{L-} n}$ and $b_{n}=$ $b_{R+} e^{i \mu_{R} q_{R+} n}+b_{R-} e^{i \mu_{R} q_{R-} n}$, respectively, where $q_{L(R) \pm}$ is determined by the solution of the eigenvalue equation, Eq. (20), with positive imaginary part:

$$
\cos q_{L(R) \pm}=\frac{-\omega_{0 L(R)} \cos \alpha \pm i \sqrt{\omega_{0 L(R)}^{2} \sin ^{2} \alpha-\omega^{2}}}{2 t_{L(R)}} .
$$

According to this expression, bound states can only appear at energies with $\omega^{2}<\omega_{0 L(R)}^{2} \sin ^{2} \alpha$, i.e., at energies within the gap formed by the Andreev bands of both ACs [cf. Eq. (19)]. The corresponding eigenvectors are given by

$$
b_{L(R) \pm}=\left(\begin{array}{c}
1 \\
i e^{ \pm i \gamma_{L(R)}}
\end{array}\right)
$$

where

$$
e^{ \pm i \gamma_{L(R)}}=\frac{-\omega \pm i \sqrt{\omega_{0 L(R)}^{2} \sin ^{2} \alpha-\omega^{2}}}{\omega_{0 L(R)} \sin \alpha} .
$$

From the above results we find that bound states exist for those energies satisfying following determinant equation:

$$
\left|\begin{array}{cccc}
t_{L} & t_{L} & t_{R} & t_{R} \\
t_{L} e^{i \gamma_{L}} & t_{L} e^{-i \gamma_{L}} & t_{R} e^{i \gamma_{R}} & t_{R} e^{-i \gamma_{R}} \\
e^{i q_{L+}} & e^{i q_{L-}} & e^{-i q_{R+}} & e^{-i q_{R-}} \\
e^{i q_{L+}} e^{i \gamma_{L}} & e^{i q_{L-}} e^{-i \gamma_{L}} & e^{-i q_{R+}} e^{i \gamma_{R}} & e^{-i q_{R-}} e^{-i \gamma_{R}}
\end{array}\right|=0 .
$$

One can check that this equation has solutions only when $\operatorname{sign}\left(\omega_{0 L}\right)=-\operatorname{sign}\left(\omega_{0 R}\right)$. Therefore, the bound states can only appear in junctions between ACs with inverted gaps. This is a necessary but not sufficient condition. Namely, the presence of the interfacial state in inverted junctions depends on the magnetic rotation along the crystal described by $\alpha$ : whereas for antiferromagnetic alignment of the impurities $(\cos \alpha=0)$ the interfacial state appears in any inverted junction, for ferromagnetic ACs $(\sin \alpha=0)$ it never does. For any other value of $\alpha$ the existence of the bound state depends on $\Phi_{L}$ and $\Phi_{R}$ as explained below.

The determinant equation, Eq. (24), can be reduced to a compact equation in the antisymmetric configuration with $\Phi_{R}=-\Phi_{L}$. In this situation we can define $\gamma \equiv \gamma_{L}=\gamma_{R}$ and $q_{L \pm}=q_{R \mp} \equiv \pm \kappa+i \lambda$, where $\kappa$ and $\lambda$ are real numbers determined by Eq.(21). For $\lambda>0$, the condition for the existence of the bound state reads

$$
\sin ^{2} \gamma \cosh ^{2} \lambda-\sin ^{2} \kappa=0 .
$$

In Fig. 3, we show the dependence of the positive energy bound states with $\alpha$ in antisymmetric inverted junctions of ACs with fixed value of $a=2 \xi_{0}$ and different strengths of the magnetic impurities, $\Phi \equiv \Phi_{R}=-\Phi_{L}$. With shaded areas we show the energies within the positive-energy Andreev band is situated in the infinite AC [Eq. (19)]. The dotted lines correspond to energy values for which $\omega^{2}=\omega_{0}^{2} \sin ^{2} \alpha$ and they indicate the maximum possible energy of a bound state [Eq. (23)]. Close to the gap-closing point, $\cos \Phi \ll 1$, the interfacial states are present at any value of $\alpha$, excluding ferromagnetic ordering of the impurities, $\sin \alpha=0$. As the size of the gap between the Andreev bands increases, the range of $\alpha$ values for which the pair of bound states exist shrinks around those values corresponding to an antiferromagnetic ordering of the magnetic impurities, $\cos \alpha=0$.

The existence or not of the bound state in anti-symmetric junctions of ACs can be understood from the relative position of the maximum-energy condition for the bound state (the dotted lines in Fig. 3) and the positive-energy solution of Eq. (25). When $\cos \alpha \approx 0$ the maximum-energy condition locates very 


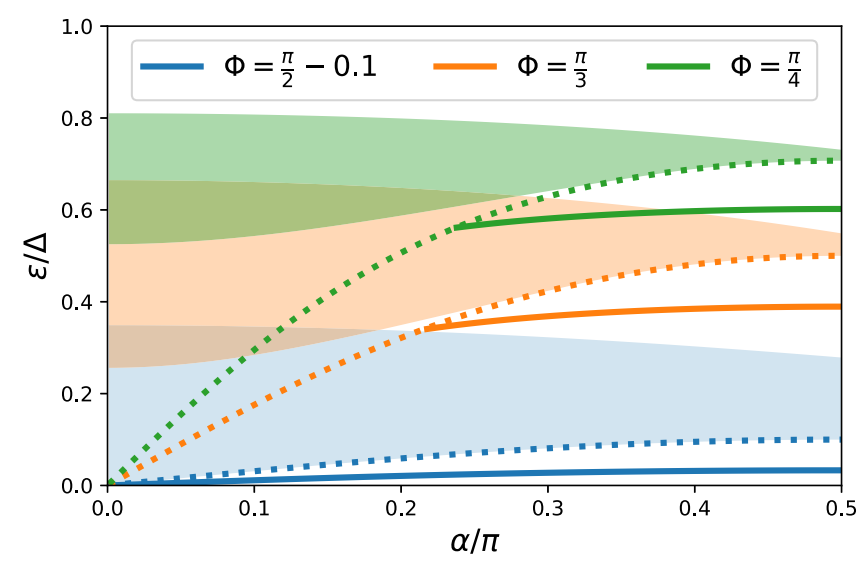

FIG. 3. Energy of the (solid line) positive-energy interfacial state in terms of $\alpha$ in anti-symmetric junctions between helical ACs with $\Phi \equiv \Phi_{R}=-\Phi_{L}$. Different colors correspond to different strengths of the impurities, $\Phi$, whereas their separation all along the junction is fixed to $a=2 \xi_{0}$. The shaded areas indicate the position of the (positive-energy) Andreev band in the respective infinite chains [Eq. (19)] and the dotted lines show the energy values with $\omega^{2}=$ $\omega_{0}^{2} \sin ^{2} \alpha$. This value determines the maximum possible energy of the bound state [Eq. (23)].

close to the bottom of the Andreev band and, consequently, the $\omega$ value that solves Eq. (25) almost always fulfills that $\omega^{2}<\omega_{0}^{2} \sin ^{2} \alpha$. When $\sin \alpha \approx 0$, by contrast, the dotted lines in Fig. 3 approach the center of the gap, $\omega=0$. Thus the solution to Eq. (25) only meets the bound state existence condition, $\omega^{2}<\omega_{0}^{2} \sin ^{2} \alpha$, when the borders of the gap are also very close to the Fermi energy, i.e., when $\cos \Phi \approx 0$. These considerations are also applicable in general junctions between helical ACs, in which case the energy of the bound state solves Eq. (24) and its existence condition is given by $\omega^{2}<\min \left(\omega_{0 L}^{2}, \omega_{0 R}^{2}\right) \sin ^{2} \alpha$.

As a summary of this section, for a given value of the rotation angle $\alpha$ we can classify ACs in two groups depending on whether an interfacial state appears upon the formation of a junction between two chains with inverted gaps. These two groups are best exemplified by (anti)ferromagnetic ACs inverted junctions in which interfacial bound states (always) never appear. In the next section, we focus on these two type of junctions and study in more detail their spatial properties.

\section{COLLINEAR ANDREEV CRYSTALS}

In this section, we extend the study of (anti-)ferromagnetic ACs beyond the first neighbor tight-binding approximation used in previous sections. To do so, we solve the Eilenberger equation to obtain the quasiclassical GFs, $\breve{g}(x)$, following the procedure discussed in Sec. II B. From the knowledge of $\breve{g}(x)$, we can obtain the local density of states and magnetization of $\mathrm{ACs}$ and junctions.

Specifically, we consider chains of magnetic impurities located at $X_{n}=n a$, with an arbitrary separation between the impurities $a$. Here, $n$ is an integer. We assume that all magnetizations, and hence the exchange fields, are aligned along the $z$ axis. Because of the collinear alignment of the exchange field we can treat the two spin degrees of freedom separately, $\sigma= \pm$, thus reducing the size of the GFs involved from $4 \times 4$ (in Nambu $\times$ spin space) to $2 \times 2$ matrices in Nambu space. It follows from Eq. (10) and the normalization condition, $[\check{g}(x)]^{2}=1$, that within the region between two subsequent impurities, $X_{n}<x<X_{n+1}$, the quasiclassical GF for a single spin projection, $\sigma$, can be written in terms of two independent constants, $b_{\sigma n}$ and $c_{\sigma n}$ :

$$
\begin{aligned}
\hat{g}_{\sigma}(x)= & \sqrt{1-e^{-2 a / \xi} b_{\sigma n} c_{\sigma n}} \hat{g}^{0}+b_{\sigma n} e^{2\left(x-X_{n+1}\right) / \xi}|+\rangle\langle\tilde{-}| \\
& +c_{\sigma n} e^{-2\left(x-X_{n}\right) / \xi}|-\rangle\langle\tilde{+}| .
\end{aligned}
$$

Here $\hat{g}^{0} \equiv \hat{P}_{+}-\hat{P}_{-}=\frac{\Delta \hat{\tau}_{2}+i \epsilon \hat{\tau}_{3}}{\sqrt{\Delta^{2}-\epsilon^{2}}}$ is the GF of an homogeneous BCS superconductor. Equation (26) is the representation of the GF in the basis where the BCS propagator, Eq. (11), is diagonal.

According to Eq. (13), the GFs at the left and right sides of the $n$th impurity are connected by the boundary condition

$$
\hat{g}_{\sigma}\left(X_{n}^{R}\right)=e^{i \sigma \hat{\tau}_{3} \Phi_{n}} \hat{g}_{\sigma}\left(X_{n}^{L}\right) e^{-i \sigma \hat{\tau}_{3} \Phi_{n}},
$$

where the direction to which the exchange field is pointing along the quantization axis is determined by the sign of the magnetic phase $\Phi_{n}$. Ferromagnetic ACs are described by a sequence of identical magnetic impurities with associated magnetic phases of $\Phi_{n}=\Phi$, whereas in antiferromagnetic ACs $\Phi_{n}=(-1)^{n} \Phi$. In the next sections, we study these two types of ACs and junctions between them.

\section{A. Ferromagnetic ACs}

In a ferromagnetic $\mathrm{AC}$, the unit cell contains a single magnetic impurity, so the $\sigma$-spin projection of the chain propagator, $\mathrm{Eq}(14)$, reads

$$
\hat{S}_{F \sigma} \equiv \hat{u}(a) e^{i \sigma \hat{\tau}_{3} \Phi} .
$$

Here $\hat{u}(a)$ is the BCS propagator given in Eq. (11). The operator $\hat{S}_{F \sigma}$ describes the propagation of the quasiclassical GF from the left side of impurity $n$ to the left side of impurity $n+1, \hat{g}_{\sigma}\left(X_{n+1}^{L}\right)=\hat{S}_{F \sigma} g_{\sigma}\left(X_{n}^{L}\right) \hat{S}_{F \sigma}^{-1}$.

To determine the quasiclassical GF, we need to obtain the parameters $b$ and $c$ in Eq. (26). The periodicity of $\hat{g}_{\sigma}$ over the unit cell, leads to $b_{\sigma n}=b_{\sigma}$ and $c_{\sigma n}=c_{\sigma}$. These expressions together with $\hat{S}_{F \sigma} \hat{g}\left(X_{n}^{L}\right) \hat{S}_{F \sigma}^{-1}=\hat{g}\left(X_{n}^{L}\right)$ result in

$$
b_{\sigma}=c_{\sigma}=\frac{e^{\frac{a}{\xi}}\left\langle\tilde{f}\left|e^{i \sigma \hat{\tau}_{3} \Phi}\right|-\right\rangle}{\left.\sqrt{\left(\frac{e^{\frac{a}{\xi}}\left\langle\tilde{f}\left|e^{i \sigma \hat{\tau}_{3} \Phi}\right|+\right\rangle+e^{-\frac{a}{\xi}}}{2}\left\langle\tilde{\tau}\left|e^{i \sigma \hat{\tau}_{3} \Phi}\right|-\right\rangle\right.}\right)^{2}-1} .
$$

After substitution of these values in Eq. (26) one obtains the quasiclassical GF in the magnetic regions all along the chain and, with it, the local density of states (LDOS) and the local spin density [Eqs. (15) and (16), respectively].

In Fig. 4, we show the LDOS for a single spin species of different ferromagnetic ACs, $v_{\uparrow}(\epsilon)$. The LDOS of the opposite spin species can be obtained from the relation $\nu_{\downarrow}(\epsilon)=$ $\nu_{\uparrow}(-\epsilon)$. The different panels in Fig. 4, correspond to different values of separation and strength of the magnetic impurities, $a$ and $\Phi$, respectively. Within the superconducting gap, $|\epsilon|<$ $|\Delta|$, the position of the Andreev band depends on $\Phi$ and its 

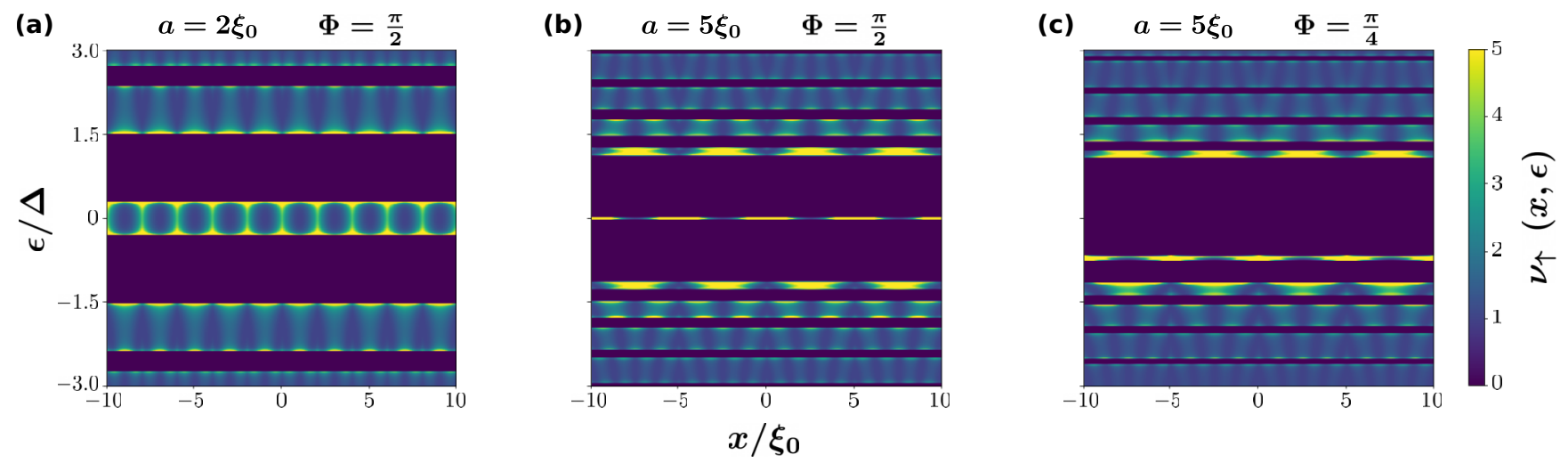

FIG. 4. Local density of states (LDOS), $v_{\uparrow}$, of spin-up quasiparticles in ferromagnetic ACs with different separation between and strengths of the magnetic impurities, $a$ and $\Phi$, respectively (see the title above each panel). A spin-polarized Andreev band, whose width increases with decreasing $a$, moves from the lower edge of the superconducting gap to the top one with increasing value of $\Phi$, crossing zero energy around $\sin \Phi=0$ values. The LDOS of spin-down quasiparticles fulfills the relation $v_{\downarrow}(\epsilon)=\nu_{\uparrow}(-\epsilon)$.

width increases by decreasing $a$. For energies larger than $\Delta$ the continuum gets split by small gaps whose widths depend on $\Phi, a$ and the energy at which they lay. The origin of the gaps lay on the lifting of degeneracies between electronic states that differ by the reciprocal lattice vector in periodic crystals, studied in many textbooks [44-46]. At integer values of $\Phi / \pi$, the small gaps at the continuum close, whereas their width is maximum for half-integer values of $\Phi / \pi$. In the same way as it happens with the Andreev band, the width of these small gaps increases with decreasing $a$. The size of the gaps reduces by increasing the energy with respect to the Fermi level.

\section{B. Antiferromagnetic ACs}

In antiferromagnetic ACs, the unit cell contains two identical magnetic impurities pointing in opposite directions. The chain propagator [Eq. (14)] that describes the evolution of the GF from the left interface of one magnetic impurity to the left interface of the equivalent impurity in the next unit cell reads,

$$
\hat{S}_{A \sigma} \equiv \hat{u}(a) e^{-i \sigma \hat{\tau}_{3} \Phi} \hat{u}(a) e^{i \sigma \hat{\tau}_{3} \Phi} .
$$

The unit cell consists now of two superconducting regions with two different sets of independent parameters, namely, $b_{\sigma 0}=b_{\sigma(2 n)}, c_{\sigma 0}=c_{\sigma(2 n)}$ and $b_{\sigma 1}=b_{\sigma(2 n+1)}, c_{\sigma 1}=c_{\sigma(2 n+1)}$. Here $n$ is the impurity index. The boundary condition for the impurity located between these two superconducting sections, Eq. (27), leads to the following relation between the set of parameters:

$$
b_{\sigma 1}=c_{\sigma 0}, \quad c_{\sigma 1}=b_{\sigma 0} .
$$

Additionally, from the periodicity of the GF, $\hat{S}_{A \sigma} \hat{g}_{\sigma}\left(X_{2 n}^{L}\right) \hat{S}_{A \sigma}^{-1}=\hat{g}_{\sigma}\left(X_{2 n}^{L}\right)$, we obtain the expressions for

$$
\begin{gathered}
b_{\sigma 0}=\left\langle\tilde{-}\left|e^{i \sigma \hat{\tau}_{3} \Phi}\right|-\right\rangle \mathcal{D}_{\sigma}, \\
c_{\sigma 0}=-\left\langle\tilde{+}\left|e^{i \sigma \hat{\imath}_{3} \Phi}\right|+\right\rangle \mathcal{D}_{\sigma},
\end{gathered}
$$

where

$$
\mathcal{D}_{\sigma} \equiv \frac{e^{\frac{a}{\xi}}\left\langle\tilde{f}\left|e^{i \sigma \hat{\imath}_{3} \Phi}\right|-\right\rangle}{\sqrt{\left\langle\tilde{+}\left|e^{i \sigma \hat{\imath}_{3} \Phi}\right|+\right\rangle\left\langle\tilde{-}\left|e^{i \sigma \hat{\imath}_{3} \Phi}\right|-\right\rangle+\left(\left\langle\tilde{+}\left|e^{i \sigma \hat{\tau}_{3} \Phi}\right|+\right\rangle\left\langle\tilde{-}\left|e^{i \sigma \hat{\imath}_{3} \Phi}\right|-\right\rangle \sinh \frac{a}{\xi}\right)^{2}}} .
$$

Substitution of these expressions into Eq. (26) determines the quasiclassical GF. From it we obtain the LDOS for a single spin specie shown in Fig. 5 for different values of $\Phi$ around the gap closing point, $\Phi=\pi / 2$. The separation between impurities is set to $a=2 \xi_{0}$. For energies within the superconducting gap, a pair of Andreev bands appear at symmetric energy ranges with respect to the Fermi level. As it was predicted in previous calculations under the first-neighbor tight-binding approximation (Ref. [23] and Sec. III), these two bands touch each other only at half-integer values of $\Phi / \pi$ closing the gap around the Fermi level (see Fig. 5). Moreover, the Andreev bands touch the continuum only when $\Phi / \pi$ is an integer: situations where the LDOS of the antiferromagnetic $\mathrm{AC}$ coincides with that of a pristine superconductor because the phase difference obtained by electrons and holes after propagation across an impurity is a multiple of $2 \pi$. Within the Andreev bands, the LDOS is larger around the position of the magnetic impurities and around the energies of the singleimpurity level. For energies larger than the superconducting gap, $|\epsilon|>|\Delta|$, we observe an interference pattern and the splitting of the continuum due to the opening of small gaps. The dependence of the width of the small gaps on $\Phi, a$, and $\epsilon$ is the same as the one observed in ferromagnetic ACs (see the last paragraph of Sec. IV A). 

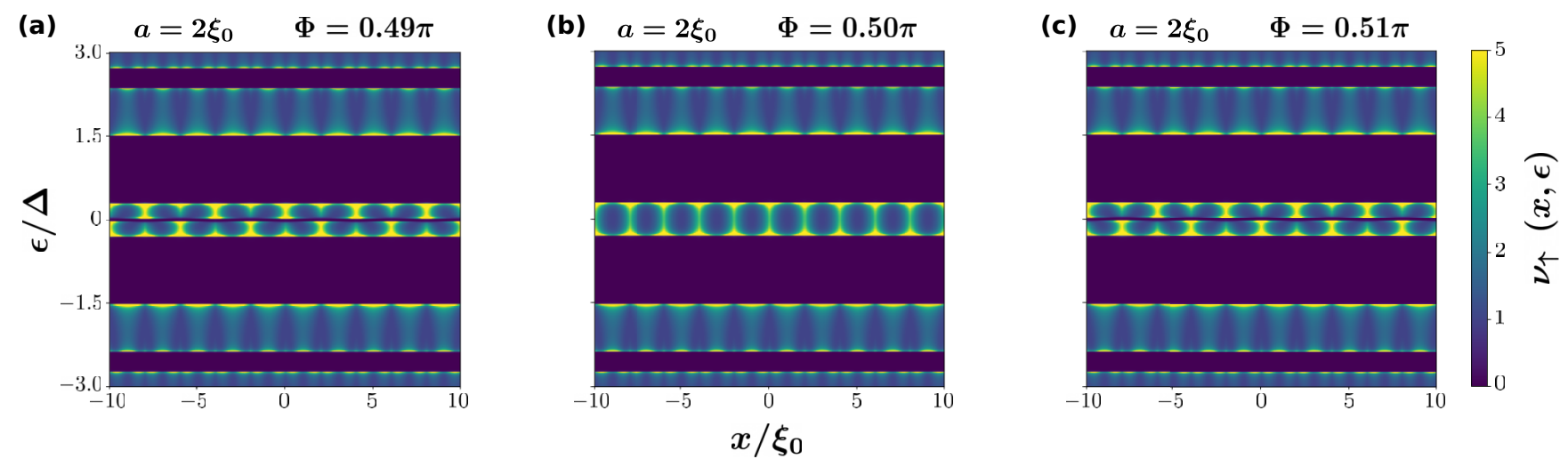

FIG. 5. Local density of states, $v_{\uparrow}$, of spin-up electrons in an antiferromagnetic AC close to the gap closing event, cos $\Phi \ll 1$. The separation between impurities is $a=2 \xi_{0}$ and different panels correspond to different values of $\Phi$. The gap between the Andreev bands closes only at values of $\cos \Phi=0$.

\section{Junctions of collinear ACs}

As discussed in Sec. III, inverted junctions of antiferromagnetic ACs host a pair of states bounded to the interface. Moreover, inverted junctions of antiferromagnetic ACs may present fractionalization of the surface spin polarization per Fermi valley[23]. In this section we show that this result holds beyond the tight-binding approximation used above, by solving the Eilenberger equation in junctions of ACs. Although we focus our analysis on junctions between antiferromagnetic ACs, the mathematical procedure presented here is general and it can be applied to obtain the quasiclassical GFs in junctions between any type of collinear ACs.

We start by defining the $\sigma$-spin projection of the chain propagators of the left(right) ACs, $\hat{S}_{L(R) \sigma}$, as the operator that propagates the GFs through a unit cell of the crystal, Eq. (14). The chain propagator is given by Eq. (28) in ferromagnetic and by Eq. (30) in antiferromagnetic ACs. Solving the eigenvalue problem of these operators we find a set of vectors for which the chain propagator is diagonal,

$$
\hat{S}_{L(R) \sigma}\left|\lambda_{L(R) \sigma}^{ \pm}\right\rangle=e^{ \pm \lambda_{L(R) \sigma}}\left|\lambda_{L(R) \sigma}^{ \pm}\right\rangle .
$$

Because $\hat{S}_{L(R) \sigma}$ is, in general, not Hermitian, the left eigenvectors that form the co-basis

$$
\left\langle\tilde{\lambda}_{L(R) \sigma}^{ \pm}\right| \hat{S}_{L(R) \sigma}=e^{ \pm \lambda_{L(R) \sigma}}\left\langle\tilde{\lambda}_{L(R) \sigma}^{ \pm}\right|,
$$

are not related by Hermitian conjugation to the right eigenvectors in Eq. (35). The eigenvectors can be represented as exponentials with arguments of opposite sign because $\operatorname{det}\left(\hat{S}_{L(R) \sigma}\right)=1$. In ferromagnetic and antiferromagnetic ACs, $\lambda_{\sigma}$ is purely imaginary (real) for energies where the infinite chain's spectrum shows (does not show) states. Similarly to the description of the propagation within the superconducting region between two subsequent impurities, Eq. (10), the propagation of the spin-polarized GFs through the reference points of different unit cells reads

$$
\begin{aligned}
\hat{g}_{\sigma}(m l)= & \sqrt{1-v_{s \sigma} w_{s \sigma}}\left(\left|\lambda_{s \sigma}^{+}\right\rangle\left\langle\tilde{\lambda}_{s \sigma}^{+}|-| \lambda_{s \sigma}^{-}\right\rangle\left\langle\tilde{\lambda}_{s \sigma}^{-}\right|\right) \\
& +v_{s \sigma} e^{2 \lambda_{s \sigma} m}\left|\lambda_{s \sigma}^{+}\right\rangle\left\langle\tilde{\lambda}_{s \sigma}^{-}\left|+w_{s \sigma} e^{-2 \lambda_{s \sigma} m}\right| \lambda_{s \sigma}^{-}\right\rangle\left\langle\tilde{\lambda}_{s \sigma}^{+}\right| .
\end{aligned}
$$

Here $s$ is substituted by $L$ and $R$ on the left and right ACs, respectively, $l$ is the length of the unit cell, $m$ is the unit cell index and we set the reference point inside the unit cell to $x_{0}=0$. The square root multiplying the first term on the righthand side (r.h.s.) of Eq. (37) comes from the normalization condition of the GF and the substraction of projectors that it multiplies corresponds to the quasiclassical GF of the infinite $\mathrm{AC}$ at the left interface of the reference impurity.

Equation (37) provides the quasiclassical GFs for a single spin, $\sigma$, at the reference points of each unit cell in terms of four parameters (two parameters per side of the junction): $v_{s \sigma}$ and $w_{s \sigma}$. Commensurability of $\breve{g}(x)$ at $x \rightarrow \pm \infty$ requires that at each side of the junction one of these parameters has to be zero. Which one of the parameters is set to zero depends on the sign of $\lambda_{s \sigma}$ : for $s=L(s=R)$, we set $w_{s \sigma}=0$ $\left(v_{s \sigma}=0\right)$ when $\lambda_{s \sigma}>0$, whereas we set $v_{s \sigma}=0\left(w_{s \sigma}=0\right)$ otherwise. The value of the remaining two parameters is obtained from the continuity of the quasiclassical GFs through the junction. Having obtained the four parameters we next propagate $\hat{g}_{\sigma}(\mathrm{ml})$ according to Eqs. (10) and (13) to obtain the quasiclassical GFs in any position of the chain, $x$. This method leads to analytic expressions of the quasiclassical GFs for any junction configuration. In particular, in App. A we apply this method in junctions between antiferromagnetic ACs and obtain the analytic expression of the quasiclassical GF, $\breve{g}(x)$ [Eqs. (A9)-(A18)].

In Fig. 6, we show the obtained LDOS for a single spin species around the interface between two antiferromagnetic ACs for different values of $\Phi_{L}$ and fixed values of $\Phi_{R}=0.4 \pi$ and $a=\xi_{0}$. The left panel of Fig. 6 shows the situation where the function of the energy of the single-impurity Andreev states, $\omega_{0}$, has the same sign at both sides of the junction. The spectrum exhibits a transition area around the interface where the size of the gap between the Andreev bands changes, but no bound states appear. When $\Phi_{L}=\pi / 2$ (middle panel of Fig. 6) the gap on the left side of the junction closes, whereas the gap on the right remains open. Further increasing of $\Phi_{L}$ leads to a reopening of the left gap, as shown on the right panel of Fig. 6. One can clearly see how spin-polarized bound states appear around the interface as a consequence of the gap inversion. Interestingly, these bound states are not restricted to the gap between the low-energy Andreev bands, but appear 

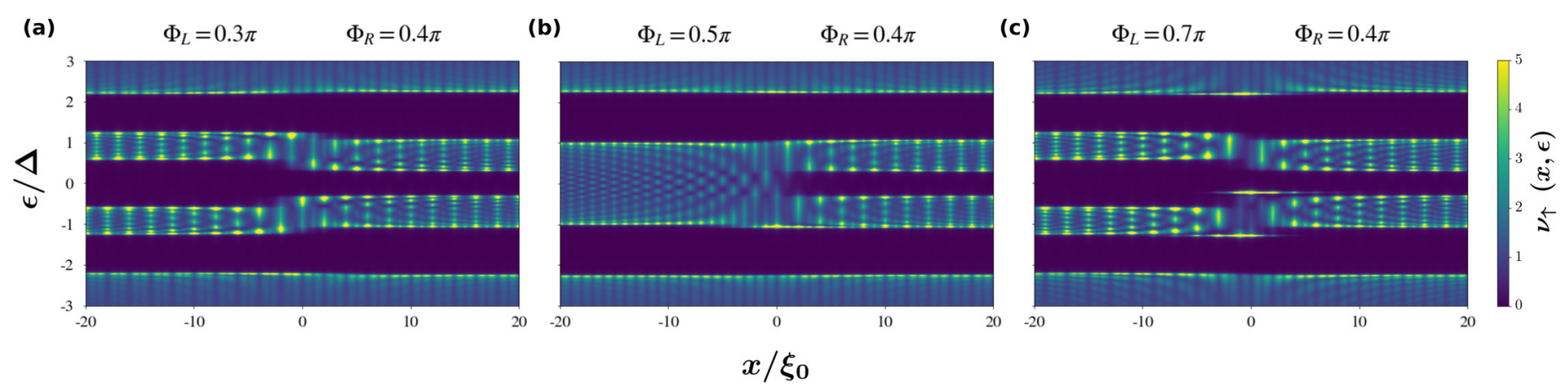

FIG. 6. Local density of states of spin-up electrons, $v_{\uparrow}(x, \epsilon)$, in a junction between two different antiferromagnetic ACs. Inversion of the gap across the junction leads to the appearance of states bounded to the interface at every gap in the spectrum. These states move from one edge of the gap to the opposite one with increasing $\Phi_{L}$. The closer the energy of the states are to the gap edge of one chain, the more they penetrate into that chain.

inside all gaps in the spectrum, indicating that the inversion of the central gap carry the invertion of all the remaining gaps.

From the quasiclassical GF of the junction, we can also compute the spin of the system by integrating Eq. (16) over $x$. We consider the zero-temperature case. As it is well know, quasiclassical GFs only describes the physics close to the Fermi surface and, hence, to obtain the total spin density one has to add the Pauli paramagnetic term [45,47]. Namely, the Pauli paramagnetic contribution of each magnetic impurity is given by $\Phi / \pi$ in units of $\hbar / 2$ [12]. The resulting total value depends on the way the ACs terminate. As we are dealing with an infinite system, it is calculated from the average over all possible ending configurations of the chains [23]. This is equivalent to the so-called sliding window average method (see, for example, Sec. 4.5 of Ref. [48]) and it results in a Pauli paramagnetic contribution of $\frac{\Phi_{L}-\Phi_{R}}{2 \pi}$ that has to be added to the integrated magnetization density of Eq. (16).

In Fig. 7, we show the contribution of a single Fermi valley to the surface spin polarization at $T=0$ of a junction between two antiferromagnetic ACs as a function of $\Phi_{L}$. We set $a=\xi_{0}$ and $\Phi_{R}=0.4 \pi$, although other values of $a$ and $-\pi / 2<\Phi_{R}<\pi / 2$ give the same results, as long as the separation between the impurities is large enough such that the regions in-between remain in the superconducting phase. The magnetization per Fermi valley can only take half-integer values of the electronic spin, which indicates fractionalization of the surface spin per electron-hole valley. The contribution

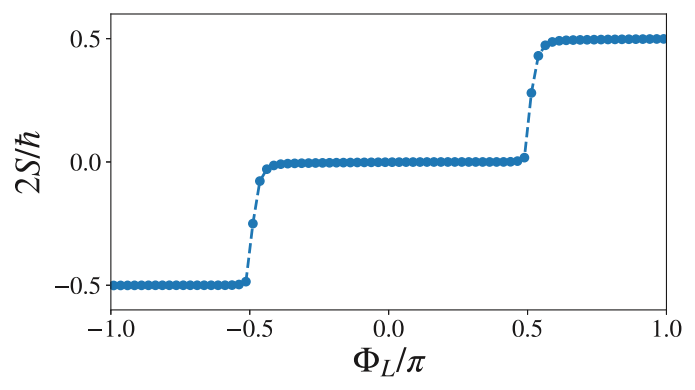

FIG. 7. Contribution of a single Fermi valley to the surface spin polarization at $T=0$ of a junction between antiferromagnetic ACs in terms of $\Phi_{L}$ for fixed values of $\Phi_{R}=0.4 \pi$ and $a=\xi_{0}$. The transition between plateaus is rounded due to the Dynes parameter, $\Gamma=10^{-3} \Delta$, used to avoid numerical convergence problems. from both Fermi valleys are equal and, hence, the total surface magnetization equals to an integer value of $\hbar / 2$. Choice of $\Phi_{R}$ outside the range $-\pi / 2<\Phi_{R}<\pi / 2$ would shift the ladderlike curve in Fig. 7 some steps up or down due to the Pauli paramagnetic contribution (see previous paragraph). Finding the value of $a$ below which superconductivity breaks down would require self-consistent calculation of $\Delta$. However, we can make an upper-bound estimation of the critical value of $a$ by demanding that the mean value of the exchange field along the wire does not exceed the value of $\Delta$. In ferromagnetic ACs this condition requires that $a>\xi_{0}$, whereas in antiferromagnetic ACs the exchange field averages to zero and superconductivity may survive even at $a<\xi_{0}$. In Fig. 7, the smooth transition between plateaus is a consequence of the small imaginary positive number that we add to the energy, $\epsilon+i \Gamma$, with $\Gamma=10^{-3} \Delta$, in order to avoid numerical problems. $\Gamma$ is known as Dynes parameter [49] and models the effect of inelastic scattering which leads to a broadening of the coherent peaks in the spectrum. In absence of inelastic processes, $\Gamma=0$ and the magnetization shows sharp steps.

\section{CONCLUSIONS}

In conclusion, we have presented an exhaustive study of ACs. We have studied the spectral properties of infinite helical $\mathrm{ACs}$ and junctions between them. For energies within the superconducting gap, the spectrum of helical ACs exhibits a pair of energy-symmetric Andreev bands with respect to the Fermi level. In ferromagnetic ACs $(\sin \alpha=0)$ the gap between the Andreev bands close in a finite range of $\Phi$ values around half-integer values of $\Phi / \pi$. The range of $\Phi$ values for which the gap remains closed increasing with decreasing separation between impurities, $a$. Otherwise, $\sin \alpha \neq 0$, the gap closes only at half-integer values of $\Phi / \pi$, forming a Dirac point. Inverted junctions of helical ACs may present a pair of states bounded to the interface. These states (always) never appear in inverted junctions of (anti)ferromagnetic ACs, whereas they more likely appear as the rotation of the ACs forming the inverted junction approaches an antiferromagnetic configuration (i.e., with decreasing value of $|\cos \alpha|$ ).

On the other hand, we show a method to solve the Eilenberger equation of infinite ACs and junctions between semi-infinite ACs. Because (anti)ferromagnetic ACs best exemplify the (existence) absence of interfacial states in inverted 
junctions between them, we apply this method to compute the full quasiclassical GFs of chains and junctions with collinear magnetization of the impurities. Our calculations are exact and generalizes the results of Ref. [23] for arbitrary distance between the impurities, namely, that the gap around the Fermi level in antiferromagnetic ACs only closes at half-integer values of $\Phi / \pi$ and that junctions between different antiferromagnetic ACs exhibit states bounded to the interface when the gap gets inverted through the junction. From the quasiclassical GFs we calculate the surface spin polarization and show that such inverted junctions show fractionalization of the surface spin. The method that we present to solve the Eilenberger equation of collinear ACs and junctions between them can be generalized for more complex magnetic configurations.

Overall, our results suggest the use of superconductorferromagnetic structures to realize crystals of a mesoscopic scale. We predict a diversity of properties of such Andreev
Crystals, as gap inversion and edge states, that can be proved by state-of-the-art spectroscopic techniques. Given the ballistic nature of the studied system, experimental observation of the predicted properties requires the use of clean materials where the mean free path is larger than the superconducting coherence length, $\ell>\xi_{0}$.

\section{ACKNOWLEDGMENTS}

M.R. and F.S.B. acknowledge funding by the Spanish Ministerio de Ciencia, Innovación y Universidades (MICINN) (Project FIS2017-82804-P), and EU's Horizon 2020 research and innovation program under Grant Agreement No. 800923 (SUPERTED). I.V.T. acknowledges support by Grupos Consolidados UPV/EHU del Gobierno Vasco (Grant No. IT1249-19).

\section{APPENDIX: QUASICLASSICAL GF IN A JUNCTION BETWEEN ANTIFERROMAGNETIC ACS}

We consider a junction between two antiferromagnetic ACs, where the separation between impurities, $a$, remains constant, but their strength changes from one chain to the other one $\left(\Phi_{L}\right.$ and $\Phi_{R}$ in the left and right AC, respectively). Both chains meet at $x=0$. The chain propagator of each chain is given by Eq. (30), substituting $\Phi$ by $\Phi_{L}$ and $\Phi_{R}$ in the left and right AC, respectively. The set of eigenvalues and left and right eigenvectors of the chain propagator in the left (right) AC that fulfill,

$$
\hat{S}_{L(R) \sigma}\left|\lambda_{L(R) \sigma}^{ \pm}\right\rangle=e^{ \pm \lambda_{L(R) \sigma}}\left|\lambda_{L(R) \sigma}^{ \pm}\right\rangle, \quad\left\langle\tilde{\lambda}_{L(R) \sigma}^{ \pm}\right| \hat{S}_{L(R) \sigma}=e^{ \pm \lambda_{L(R) \sigma}}\left\langle\tilde{\lambda}_{L(R) \sigma}^{ \pm}\right|,
$$

read

$$
\begin{aligned}
e^{ \pm \lambda_{L(R) \sigma}}= & 1+2\left\langle\tilde{+}\left|e^{i \sigma \hat{\tau}_{3} \Phi_{L(R)}}\right|+\right\rangle\left\langle\tilde{-}\left|e^{i \sigma \hat{\tau}_{3} \Phi_{L(R)}}\right|-\right\rangle \sinh ^{2} \frac{a}{\xi} \pm 2\left[\left\langle\tilde{+}\left|e^{i \sigma \hat{\tau}_{3} \Phi_{L(R)}}\right|+\right\rangle\right. \\
& \left.\times\left\langle\tilde{-}\left|e^{i \sigma \hat{\tau}_{3} \Phi_{L(R)}}\right|-\right\rangle \sinh ^{2} \frac{a}{\xi}+\left(\left\langle\tilde{+}\left|e^{i \sigma \hat{\tau}_{3} \Phi_{L(R)}}\right|+\right\rangle\left\langle\tilde{-}\left|e^{i \sigma \hat{\tau}_{3} \Phi_{L(R)}}\right|-\right\rangle \sinh ^{2} \frac{a}{\xi}\right)^{2}\right]^{1 / 2},
\end{aligned}
$$

and

$$
\left\langle\tilde{\lambda}_{L(R) \sigma}^{ \pm}\left|=c_{L(R) \sigma}^{ \pm}\left(\tilde{d}_{L(R) \sigma}^{ \pm} \quad 1\right), \quad\right| \lambda_{L(R) \sigma}^{ \pm}\right\rangle=c_{L(R) \sigma}^{ \pm}\left(\begin{array}{c}
1 \\
d_{L(R) \sigma}^{ \pm}
\end{array}\right)
$$

where

$$
\begin{gathered}
d_{L(R) \sigma}^{ \pm}=\frac{e^{ \pm \lambda_{L(R) \sigma}}-1-\left(e^{\frac{2 a}{\xi}}-1\right)\left\langle\tilde{+}\left|e^{i \sigma \hat{t}_{3} \Phi_{L(R)}}\right|+\right\rangle\left\langle\tilde{-}\left|e^{i \sigma \hat{\tau}_{3} \Phi_{L(R)}}\right|-\right\rangle}{\left(e^{\frac{2 a}{\xi}}-1\right)\left\langle\tilde{-}\left|e^{i \sigma \hat{\tau}_{3} \Phi_{L(R)}}\right|-\right\rangle\left\langle\tilde{+}\left|e^{i \sigma \hat{\tau}_{3} \Phi_{L(R)}}\right|-\right\rangle}, \\
\tilde{d}_{L(R) \sigma}^{ \pm}=\frac{e^{ \pm \lambda_{L(R) \sigma}}-1+\left(1-e^{-\frac{2 a}{\xi}}\right)\left\langle\tilde{+}\left|e^{i \sigma \hat{\tau}_{3} \Phi_{L(R)}}\right|+\right\rangle\left\langle\tilde{-}\left|e^{i \sigma \hat{\tau}_{3} \Phi_{L(R)}}\right|-\right\rangle}{\left(e^{\frac{2 a}{\xi}}-1\right)\left\langle\tilde{-}\left|e^{i \sigma \hat{\tau}_{3} \Phi_{L(R)}}\right|-\right\rangle\left\langle\tilde{+}\left|e^{i \sigma \hat{\tau}_{3} \Phi_{L(R)}}\right|-\right\rangle} \\
c_{L(R) \sigma}^{ \pm}=\sqrt{\frac{\left(e^{\frac{2 a}{\xi}}-1\right)\left\langle\tilde{-}\left|e^{i \sigma \hat{\tau}_{3} \Phi_{L(R)}}\right|-\right\rangle\left\langle\tilde{+}\left|e^{i \sigma \hat{\tau}_{3} \Phi_{L(R)}}\right|-\right\rangle}{2 \sinh \lambda_{L(R) \sigma}}} .
\end{gathered}
$$

Here $\xi=\frac{\hbar v_{F}}{\sqrt{\Delta^{2}-\epsilon^{2}}}$ is the energy-dependent superconducting coherence length. Note that $\tilde{d}_{L(R) \sigma}^{ \pm}=d_{L(R) \sigma}^{\mp}$.

We can parametrize the value of the quasiclassical GF at the equivalent points of the chain in terms of the eigenvectors of the chain propagator, Eq. (A3), as follows:

$$
\hat{g}_{\sigma}\left(X_{2 m}^{L}\right)=\sqrt{1-v_{s \sigma} w_{s \sigma}}\left(\left|\lambda_{s \sigma}^{+}\right\rangle\left\langle\tilde{\lambda}_{s \sigma}^{+}|-| \lambda_{s \sigma}^{-}\right\rangle\left\langle\tilde{\lambda}_{s \sigma}^{-}\right|\right)+v_{s \sigma} e^{2 \lambda_{s \sigma} m}\left|\lambda_{s \sigma}^{+}\right\rangle\left\langle\tilde{\lambda}_{s \sigma}^{-}\left|+w_{s \sigma} e^{-2 \lambda_{s \sigma} m}\right| \lambda_{s \sigma}^{-}\right\rangle\left\langle\tilde{\lambda}_{s \sigma}^{+}\right| .
$$

Here, $m$ is the unit cell index, $X_{2 m}^{L}$ stands for the left interface of the magnetic impurity located at $X_{2 m}=2 m a$ and the subindex $s$ label the left (L) and right (R) crystal. The unit cells forming the left and right AC are those labeled by $n \leqslant 0$ and $m>0$, respectively.

For energies at which $\left|e^{ \pm \lambda_{L(R) \sigma}}\right|=1$, Eq. (A7) describes modes that propagate all along the structure. Otherwise, it describes exponentially decaying states by setting either $v_{L(R) \sigma}$ or $w_{L(R) \sigma}$ to zero to ensure commensurability of $\hat{g}_{\sigma}$ at the infinities. Which one is set to zero depends on whether $\left|e^{ \pm \lambda_{L(R) \sigma}}\right|>1$ or $\left|e^{ \pm \lambda_{L(R) \sigma}}\right|<1$. Indeed, numerical analysis of Eq. (A2) shows that 
$\left|e^{ \pm \lambda_{L(R) \sigma}}\right| \leqslant 1$ and, therefore, we can set $v_{L \sigma}=0$ and $w_{R \sigma}=0$. To obtain the remaining two parameters, we require continuity of Eq. (A7) across the junction, which yields

$$
w_{L \sigma}=2 i \frac{d_{R \sigma}^{+}-d_{L \sigma}^{+}}{d_{R \sigma}^{+}-d_{L \sigma}^{-}}, \quad v_{R \sigma}=2 i \frac{d_{R \sigma}^{-}-d_{L \sigma}^{-}}{d_{R \sigma}^{+}-d_{L \sigma}^{-}} .
$$

Here $d_{L(R) \sigma}^{ \pm}$is given by Eq. (A4).

Substituting Eq. (A8) into Eq. (A7), we get the value of the quasiclassical GF at the left interface of every second magnetic impurity, $X_{2 n}^{L}$. To obtain $\hat{g}_{\sigma}(x)$ at every point inside the unit cell, hence, we have to propagate it from $X_{2 n}^{L}$ to $x$ by means of the BCS propagator, Eq. (10), when the propagation is across the superconducting regions, and the propagation-like boundary conditions, (13), to connect the GFs at the left and right interfaces of each impurity. Such a propagation allows us writing the quasiclassical GF all along the space as follows:

$$
\hat{g}_{\sigma}(x)=\left\{\begin{array}{r}
\mathcal{B}_{m \sigma}^{0}(|-\rangle\langle\tilde{-}|-|+\rangle\langle\tilde{+}|)+\mathcal{B}_{m \sigma}^{+-} e^{2 \frac{x-X_{2 m-1}}{\xi}}|+\rangle\left\langle\tilde{-}\left|+\mathcal{B}_{m \sigma}^{-+} e^{-2 \frac{x-X_{2 m-1}}{\xi}}\right|-\right\rangle\langle\tilde{+}| \\
\text { if } X_{2 n-2}<x<X_{2 n-1}, \\
\mathcal{A}_{m \sigma}^{0}(|-\rangle\langle\tilde{-}|-|+\rangle\langle\tilde{+}|)+\mathcal{A}_{m \sigma}^{+-} e^{2 \frac{x-X_{2 m}}{\xi}}|+\rangle\left\langle\tilde{-}\left|+\mathcal{A}_{m \sigma}^{-+} e^{-2 \frac{x-X_{2 m}}{\xi}}\right|-\right\rangle\langle\tilde{+}| \\
\text { if } X_{2 n-1}<x<X_{2 n},
\end{array},\right.
$$

where $| \pm\rangle$ and $\langle\underline{ \pm}|$ are the right- and left-eigenvectors of the BCS propagator given by Eqs. (5) and (12), respectively. The expressions of the remaining constants depend on the side of the juction. The $\mathcal{A}$ constants in the AC on the left $(m \leqslant 0)$ read

$$
\begin{gathered}
\mathcal{A}_{m \sigma}^{0}=\left(c_{L \sigma}^{+}\right)^{2}\left[d_{L \sigma}^{+}+d_{L \sigma}^{-}+i w_{L \sigma} e^{-2 \lambda_{L \sigma} m} d_{L \sigma}^{-}\right], \\
\mathcal{A}_{m \sigma}^{+-}=\left(c_{L \sigma}^{+}\right)^{2}\left[2+i w_{L \sigma} e^{-2 \lambda_{L \sigma} n}\right], \\
\mathcal{A}_{m \sigma}^{-+}=-\left(c_{L \sigma}^{+}\right)^{2} d_{L \sigma}^{-}\left[2 d_{L \sigma}^{+}+i w_{L \sigma} e^{-2 \lambda_{L \sigma} m} d_{L \sigma}^{-}\right],
\end{gathered}
$$

whereas in the right chain $(m>0)$, they read

$$
\begin{aligned}
\mathcal{A}_{m \sigma}^{0}= & \left(c_{R \sigma}^{+}\right)^{2}\left[d_{R \sigma}^{+}+d_{R \sigma}^{-}+i v_{R \sigma} e^{2 \lambda_{R \sigma} m} d_{R \sigma}^{+}\right], \\
& \mathcal{A}_{m \sigma}^{+-}=\left(c_{R \sigma}^{+}\right)^{2}\left[2+i v_{R \sigma} e^{2 \lambda_{R \sigma} n}\right], \\
\mathcal{A}_{m \sigma}^{-+}= & -\left(c_{R \sigma}^{+}\right)^{2} d_{R \sigma}^{+}\left[2 d_{R \sigma}^{-}+i v_{R \sigma} e^{2 \lambda_{R \sigma} m} d_{R \sigma}^{+}\right] .
\end{aligned}
$$

The remaining expressions for the $\mathcal{B}$-s are given in terms of the $\mathcal{A}$-s shown in Eqs. (A10)-(A15) and read

$$
\begin{gathered}
\mathcal{B}_{m \sigma}^{0}=\left(1+2\left\langle\tilde{+}\left|e^{i \sigma \hat{\imath}_{3} \Phi_{s}}\right|-\right\rangle\right) \mathcal{A}_{m \sigma}^{0}+e^{-\frac{2 a}{\xi}}\left\langle\tilde{+}\left|e^{i \sigma \hat{\tau}_{3} \Phi_{s}}\right|+\right\rangle\left\langle\tilde{+}\left|e^{i \sigma \hat{\tau}_{3} \Phi_{s}}\right|-\right\rangle \mathcal{A}_{m \sigma}^{+-}-e^{\frac{2 a}{\xi}}\left\langle\tilde{-}\left|e^{i \sigma \hat{\tau}_{3} \Phi_{s}}\right|-\right\rangle\left\langle\tilde{+}\left|e^{i \sigma \hat{\tau}_{3} \Phi_{s}}\right|-\right\rangle \mathcal{A}_{m \sigma}^{-+}, \\
\mathcal{B}_{m \sigma}^{+-}=2\left\langle\tilde{+}\left|e^{i \sigma \hat{\tau}_{3} \Phi_{s}}\right|+\right\rangle\left\langle\tilde{+}\left|e^{i \sigma \hat{\tau}_{3} \Phi_{s}}\right|-\right\rangle \mathcal{A}_{m \sigma}^{0}+e^{-\frac{2 a}{\xi}}\left\langle\tilde{+}\left|e^{i \sigma \hat{\tau}_{3} \Phi_{s}}\right|+\right\rangle^{2} \mathcal{A}_{m \sigma}^{+-}-e^{\frac{2 a}{\xi}}\left\langle\tilde{+}\left|e^{i \sigma \hat{\tau}_{3} \Phi_{s}}\right|-\right\rangle^{2} \mathcal{A}_{m \sigma}^{-+}, \\
\mathcal{B}_{m \sigma}^{-+}=-2\left\langle\tilde{-}\left|e^{i \sigma \hat{\tau}_{3} \Phi_{s}}\right|-\right\rangle\left\langle\tilde{+}\left|e^{i \sigma \hat{\tau}_{3} \Phi_{s}}\right|-\right\rangle \mathcal{A}_{m \sigma}^{0}-e^{-\frac{2 a}{\xi}}\left\langle\tilde{+}\left|e^{i \sigma \hat{\tau}_{3} \Phi_{s}}\right|-\right\rangle^{2} \mathcal{A}_{m \sigma}^{+-}+e^{\frac{2 a}{\xi}}\left\langle\tilde{-}\left|e^{i \sigma \hat{\tau}_{3} \Phi_{s}}\right|-\right\rangle^{2} \mathcal{A}_{m \sigma}^{-+},
\end{gathered}
$$

where $\Phi_{s}=\Phi_{L}$ when $m \leqslant 0$ (i.e., in the left side of the junction) and $\Phi_{s}=\Phi_{R}$ otherwise. Equations (A9)-(A18) provide the quasiclassical GF for the $\sigma$ spin component of a junction between two antiferromagnetic ACs at any position, $x$, from which we can directly calculate observables like the local density of states, Eq. (15), or the local spin density, Eq. (16).

[1] L. Yu, Bound state in superconductors with paramagnetic impurities, Acta Phys. Sin 21, 75 (1965).

[2] H. Shiba, Classical spins in superconductors, Prog. Theor. Phys. 40, 435 (1968).

[3] A. Rusinov, Superconductivity near a paramagnetic impurity, Zh. Eksp. Teor. Fiz. Pisma Red. 9, 146 (1968) [Sov. Phys. JETP 9, 85 (1969)]

[4] A. Andreev, Electron spectrum of the intermediate state of superconductors, Zh. Eksp. Teor. Fiz. 49, 665 (1965), [Sov. Phys. JETP 22, 455 (1966)].

[5] A. Sakurai, Comments on Superconductors with Magnetic Impurities, Prog. Theor. Phys. 44, 1472 (1970).
[6] A. Yazdani, Probing the local effects of magnetic impurities on superconductivity, Science 275, 1767 (1997).

[7] A. V. Balatsky, I. Vekhter, and J.-X. Zhu, Impurity-induced states in conventional and unconventional superconductors, Rev. Mod. Phys. 78, 373 (2006).

[8] K. J. Franke, G. Schulze, and J. I. Pascual, Competition of superconducting phenomena and kondo screening at the nanoscale, Science 332, 940 (2011).

[9] T. Meng, J. Klinovaja, S. Hoffman, P. Simon, and D. Loss, Superconducting gap renormalization around two magnetic impurities: From shiba to andreev bound states, Phys. Rev. B 92, 064503 (2015). 
[10] B. W. Heinrich, J. I. Pascual, and K. J. Franke, Single magnetic adsorbates on s-wave superconductors, Prog. Surf. Sci. 93, 1 (2018).

[11] L. Farinacci, G. Ahmadi, G. Reecht, M. Ruby, N. Bogdanoff, O. Peters, B. W. Heinrich, F. von Oppen, and K. J. Franke, Tuning the Coupling of an Individual Magnetic Impurity to a Superconductor: Quantum Phase Transition and Transport, Phys. Rev. Lett. 121, 196803 (2018).

[12] M. Rouco, I. V. Tokatly, and F. S. Bergeret, Spectral properties and quantum phase transitions in superconducting junctions with a ferromagnetic link, Phys. Rev. B 99, 094514 (2019).

[13] F. Konschelle, I. V. Tokatly, and F. S. Bergeret, Ballistic Josephson junctions in the presence of generic spin dependent fields, Phys. Rev. B 94, 014515 (2016).

[14] S. Nadj-Perge, I. K. Drozdov, B. A. Bernevig, and A. Yazdani, Proposal for realizing majorana fermions in chains of magnetic atoms on a superconductor, Phys. Rev. B 88, 020407(R) (2013).

[15] F. Pientka, L. I. Glazman, and F. von Oppen, Topological superconducting phase in helical shiba chains, Phys. Rev. B 88, 155420 (2013).

[16] A. Heimes, P. Kotetes, and G. Schön, Majorana fermions from shiba states in an antiferromagnetic chain on top of a superconductor, Phys. Rev. B 90, 060507(R) (2014).

[17] K. Pöyhönen, A. Westström, J. Röntynen, and T. Ojanen, Majorana states in helical Shiba chains and ladders, Phys. Rev. B 89, 115109 (2014).

[18] A. Westström, K. Pöyhönen, and T. Ojanen, Topological properties of helical Shiba chains with general impurity strength and hybridization, Phys. Rev. B 91, 064502 (2015).

[19] F. Pientka, Y. Peng, L. Glazman, and F. v. Oppen, Topological superconducting phase and majorana bound states in shiba chains, Phys. Scr. T164, 014008 (2015).

[20] P. M. R. Brydon, S. Das Sarma, H.-Y. Hui, and J. D. Sau, Topological Yu-Shiba-Rusinov chain from spin-orbit coupling, Phys. Rev. B 91, 064505 (2015).

[21] M. Schecter, K. Flensberg, M. H. Christensen, B. M. Andersen, and J. Paaske, Self-organized topological superconductivity in a Yu-Shiba-Rusinov chain, Phys. Rev. B 93, 140503(R) (2016)

[22] S. Hoffman, J. Klinovaja, and D. Loss, Topological phases of inhomogeneous superconductivity, Phys. Rev. B 93, 165418 (2016)

[23] M. Rouco, F. S. Bergeret, and I. V. Tokatly, Gap inversion in quasi-one-dimensional Andreev crystals, Phys. Rev. B 103, 064505 (2021).

[24] A. I. Buzdin, Proximity effects in superconductor-ferromagnet heterostructures, Rev. Mod. Phys. 77, 935 (2005).

[25] F. S. Bergeret, A. F. Volkov, and K. B. Efetov, Odd triplet superconductivity and related phenomena in superconductorferromagnet structures, Rev. Mod. Phys. 77, 1321 (2005).

[26] D. Beckmann, H. B. Weber, and H. v. Löhneysen, Evidence for Crossed Andreev Reflection in Superconductor-Ferromagnet Hybrid Structures, Phys. Rev. Lett. 93, (2004).

[27] T. S. Khaire, M. A. Khasawneh, W. Pratt Jr, and N. O. Birge, Observation of Spin-Triplet Superconductivity in Co-Based Josephson Junctions, Phys. Rev. Lett. 104, 137002 (2010).

[28] N. Banerjee, C. Smiet, R. Smits, A. Ozaeta, F. Bergeret, M. Blamire, and J. Robinson, Evidence for spin selectivity of triplet pairs in superconducting spin valves, Nat. Commun. 5, 1 (2014).

[29] A. Singh, S. Voltan, K. Lahabi, and J. Aarts, Colossal Proximity Effect in a Superconducting Triplet Spin Valve Based on the Half-Metallic Ferromagnet cro 2, Phys. Rev. X 5, 021019 (2015).

[30] J. Linder and J. W. Robinson, Superconducting spintronics, Nat. Phys. 11, 307 (2015).

[31] S. V. Bakurskiy, M. Y. Kupriyanov, A. A. Baranov, A. A. Golubov, N. V. Klenov, and I. I. Soloviev, Proximity effect in multilayer structures with alternating ferromagnetic and normal layers, JETP Lett. 102, 586 (2015).

[32] L. Gor'kov and V. Kresin, Giant magnetic effects and oscillations in antiferromagnetic josephson weak links, Appl. Phys. Lett. 78, 3657 (2001).

[33] B. M. Andersen and P. Hedegård, Andreev bound states at the interface of antiferromagnets and $d$-wave superconductors, Phys. Rev. B 66, 104515 (2002).

[34] I. V. Bobkova, P. J. Hirschfeld, and Y. S. Barash, SpinDependent Quasiparticle Reflection and Bound States at Interfaces with Itinerant Antiferromagnets, Phys. Rev. Lett. 94, 037005 (2005).

[35] B. M. Andersen, I. V. Bobkova, P. J. Hirschfeld, and Y. S. Barash, Bound states at the interface between antiferromagnets and superconductors, Phys. Rev. B 72, 184510 (2005).

[36] R. Jackiw and C. Rebbi, Solitons with fermion number, Phys. Rev. D 13, 3398 (1976).

[37] W. P. Su, J. R. Schrieffer, and A. J. Heeger, Solitons in Polyacetylene, Phys. Rev. Lett. 42, 1698 (1979).

[38] W. P. Su, J. R. Schrieffer, and A. J. Heeger, Soliton excitations in polyacetylene, Phys. Rev. B 22, 2099 (1980).

[39] B. Volkov and O. Pankratov, Two-dimensional massless electrons in an inverted contact, JETP Lett 42, 178 (1985).

[40] F. Konschelle, F. S. Bergeret, and I. V. Tokatly, Semiclassical Quantization of Spinning Quasiparticles in Ballistic Josephson Junctions, Phys. Rev. Lett. 116, 237002 (2016).

[41] P. G. de Gennes, Superconductivity of Metals and Alloys (Benjamin, New York, 1966).

[42] A. F. Andreev, The thermal conductivity of the intermediate state in superconductors, Zh. Eksp. Teor. Fiz. 46, 1823 (1964) [Sov. Phys. JETP 19, 1228 (1964)]

[43] G. Eilenberger, Transformation of gorkov's equation for type ii superconductors into transport-like equations, Zeitschrift für Physik A Hadrons and nuclei 214, 195 (1968).

[44] N. Ashcroft and D. Mermin, Solid State Physics (Cengage Learning, Andover, England, 1976).

[45] A. A. Abrikosov, Fundamentals of the Theory of Metals (Dover, New York, 2017).

[46] C. Kittel, Introduction to Solid State Physics, 8th ed. (Wiley, Hoboken, NJ, 2004).

[47] X. P. Zhang, V. N. Golovach, F. Giazotto, and F. S. Bergeret, Phase-controllable nonlocal spin polarization in proximitized nanowires, Phys. Rev. B 101, 180502(R) (2020).

[48] D. Vanderbilt, Berry Phases in Electronic Structure Theory (Cambridge University Press, Cambridge, 2018).

[49] R. C. Dynes, V. Narayanamurti, and J. P. Garno, Direct Measurement of Quasiparticle-Lifetime Broadening in a Strong-Coupled Superconductor, Phys. Rev. Lett. 41, 1509 (1978). 